\title{
Causas que influyen en el aprendizaje de los signos vitales: Elemento clave dentro del proceso enfermero
}

\author{
Rosa María Pecina Leyva
}

\begin{abstract}
RESUMEN
El propósito del presente estudio es determinar las principales causas que influyen en el aprendizaje de los signos vitales con alumnos del tercer semestre de Licenciatura en Enfermería. Se realizó un estudio descriptivo, retrospectivo y transversal con una muestra a conveniencia de 137 alumnos. Se aplicó un cuestionario cuyo análisis se realizó con estadística descriptiva. Resultados: Se encontró como factor benéfico para aprender la técnica de signos vitales las estrategias didácticas que utilizaron los profesores, pero existen múltiples causas que limitan el aprendizaje de esta actividad. El signo vital que ocasionó mayor grado de dificultad al 61\% de los alumnos fue la tensión arterial; se presentaron otros problemas en la audición (29\%), en el tacto $(21 \%)$ y durante la ejecución de la técnica de signos vitales; también se detectaron: temor (29\%) e inseguridad (27\%). El $59.2 \%$ de los alumnos expresa que no integran los resultados de los signos vitales en el proceso enfermero por falta de tiempo en los ámbitos de práctica; el $14.7 \%$ refiere que no lo hace por falta de conocimientos, y el $26.1 \%$ manifiesta que integra esos datos para identificar el diagnóstico de Enfermería. Conclusiones: Las características individuales de los alumnos originan dificultades sensitivas para llevar a cabo la técnica de signos vitales, así como la falta de destreza o práctica y la falta de tiempo durante la jornada; en las unidades hospitalarias, esto ocasiona que presenten dificultades para integrar estos parámetros en la aplicación del proceso enfermero.
\end{abstract}

Palabras clave: Aprendizaje del alumno, signos vitales, proceso enfermero.

\section{Causes that influence the learning of vital signs: A key element within the nursing process}

\begin{abstract}
Introduction: The purpose of the present study was to know causes that limit learning of the technique of the vital signs. The vital signs are the first life indicators and are necessary elements for the nursing assessment, as like an essential element of nursing caring process. Some nursing students show difficulty learning the technique of the vital signs, and there is not any research in this topic. Methodology: A research was done a research in a school consisting of human resources in nursing, transverse, retrospective and descriptive study, where 137 nursing students in second year of the career participated. Results: The students referred that the didactic strategies used by the teachers were good but the causes that limit learning the technique of the vital signs are multiple among them difficulty in the perception skills, and other causes were fear, lack of safety, etc. Conclusion: This research demonstrated that learning the technique of the vital signs due to the strategies and personal style of the nursing students.
\end{abstract}

Key words: Causes that limit learning, technique of the vital signs, nursing process.

Correspondencia: Lic. en Enf. Rosa María Pecina Leyva M.A. Universidad Autónoma de San Luis Potosí, SLP, Méx.

Niño Artillero s/n. Zona Universitaria. Tel. 4448262324 Fac. Enf.

Correo electrónico: rosypecina@yahoo.com.mx

Este artículo puede ser consultado en versión completa en http://www.medigraphic.com/enfermerianeurologica 


\section{INTRODUCCIÓN}

$\mathrm{L}$ a carrera de Enfermería es una disciplina con una gran cantidad de contenidos técnico-instrumentales, por lo que se deben buscar estrategias didácticas para enseñar habilidades que mejoren la actuación del profesional de Enfermería.

Tovar $\mathrm{B}^{1}$ expresa la necesidad de formar profesionales de Enfermería que logren aprender a conocer, aprender a hacer, aprender a vivir juntos y aprender a ser; es determinante para desarrollar las habilidades, actitudes y valores necesarios para proporcionar cuidado de enfermería de calidad.

En la profesión de Enfermería, la mayoría de las actividades que realiza el alumno se dan bajo supervisión del personal docente, por lo que se fomenta el aprendizaje tutelar o dirigido, pero a pesar de esto los alumnos presentan serias deficiencias.

Tal vez porque para el desarrollo de la práctica de Enfermería se requiere aplicar los conocimientos que conforman la base teórica del ejercicio de la Enfermería.

Uno de los conocimientos que se conceptualiza como eje invariante dentro de la currícula de la Licenciatura en Enfermería es el Cuidado Enfermero, dentro del cual se aplican contenidos teóricos o técnico-instrumentales que determinan la profesión de Enfermería y que son necesarios para proporcionar cuidado de calidad al individuo, familia y comunidad.

El Proceso del Cuidado Enfermero es el método o herramienta que nos permite brindar cuidado fundamentado científicamente; cuenta con 5 etapas: la primera es la valoración, donde se encuentra el tema de la presente investigación, el cual influye en el aprendizaje de los signos vitales.

El conocimiento de los signos vitales ayuda a la enfermera o al enfermero a utilizar el pensamiento crítico para elaborar el juicio clínico de Enfermería e identificar problemas de salud o para evaluar la respuesta del paciente a las intervenciones de Enfermería.

Para Alfaro, ${ }^{2}$ los términos pensamiento crítico, proceso enfermero y juicio clínico con frecuencia se usan como sinónimos, por lo que afirma que el proceso enfermero es un instrumento que ayuda a las enfermeras a pensar críticamente. Los principios del proceso enfermero proporcionan las bases para la toma de decisiones y apuntalan a todos los modelos enfermeros; el pensamiento crítico es la capacidad de cada persona para centrar el pensamiento en obtener los resultados que necesita. ${ }^{2}$

La inquietud por realizar este estudio parte de que la enseñanza de los signos vitales en la profesión de Licenciatura en Enfermería es uno de los conocimientos esenciales o básicos que los alumnos deben adquirir al inicio de su formación y en el que los mismos manifiestan dificultades para percibir el pulso, contarlo usando el segundero o expresan dificultad para valorar la respiración del paciente o para coordinar la percepción del pulso con el fin de valorar la tensión arterial así como para observar el manómetro que marca el parámetro o dificultad para escuchar los ruidos de Korokoff que determinan este signo vital.

La técnica de signos vitales requiere que el alumno aprenda una habilidad compleja denominada destreza perceptivomotriz, porque para realizar esta actividad necesita estrategias instruccionales organizadas y secuenciadas, con el fin de obtener mejores resultados de aprendizaje.

Davis R, ${ }^{3}$ en 1990, define las habilidades perceptivo-motoras como una serie coordinada de movimientos musculares para llevar a cabo una tarea en forma satisfactoria.

Para aprender el procedimiento perceptivo motor de los signos vitales el alumno tiene que conocer el orden en que debe ejecutarse y tomar decisiones sobre posibles alternativas de acción a seguir.

Pertinentemente, Castellanos $\mathrm{L}^{4}$ señala que los egresados de las escuelas de Enfermería tienen un desarrollo medio en la valoración de esta especialidad, por lo que sugiere necesario mejorar el proceso de formación estableciendo relaciones causa-efecto entre los resultados obtenidos y el estado de salud del paciente.

He aquí la importancia por la que la técnica de signos vitales, como parte del proceso enfermero, requiere ser enseñada explícitamente con el fin de conseguir un aprendizaje más significativo, con mayor retención y transferencia de lo aprendido.

Este trabajo pretende investigar los elementos que favorecen u obstaculizan el aprendizaje de la técnica de signos vitales tan importante en la etapa de valoración del proceso enfermero.

\section{OBJETIVO GENERAL}

Determinar las causas que influyen en el aprendizaje de los signos vitales en alumnos del Tercer Semestre de la carrera de Licenciatura en Enfermería.

\section{MARCO TEÓRICO}

Los programas académicos contemplan la práctica de Enfermería como un complemento educativo para aplicación de conocimientos, en donde el alumno aprende a resolver problemas profesionales a través de la ejecución de acciones concretas en cuya realización se pretende que el alumno integre los conocimientos teóricos. Esto ha sido confirmado por Fajardo A, ${ }^{5}$ quien enuncia que el desempeño del rol de Enfermería consiste en una serie de funciones en la atención del paciente que no forman parte de su perfil de egreso, lo 
que podría traer una falta en la ejecución de las mismas. Entre ellas podría encontrarse la enseñanza del proceso de razonamiento enfermero como parte del cuidado a la salud o las habilidades instrumentales o motrices tan comunes en la disciplina enfermera.

Según Davis, ${ }^{3}$ las actividades motrices desempeñan un papel muy importante en la vida del individuo; vivir exige cierto grado indispensable de rapidez, precisión y coordinación de movimientos.

Antes de iniciar la enseñanza de una destreza, el maestro debe considerar hasta qué punto el alumno tiene la madurez física, motriz, mental, social que requiere la misma. ${ }^{3} \mathrm{La}$ técnica de signos vitales es procedimental e implica un sinnúmero de percepciones y actividades.

Y como lo expresa Garza $R,{ }^{5}$ las personas perciben la realidad de manera distinta; las experiencias y los conocimientos previos de cada una de ellas determinan muchas veces la forma en que se ve el mundo. Y cuando se enfrentan a una situación nueva se dice que algunos la experimentan dándole mucha atención a las sensaciones mientras que otros reflexionan acerca de ella, es decir, se enfrentan a ella de un modo más racional.

Lo anterior nos permite deducir que así como existen diferentes personalidades, también hay diferentes formas de aprender, por lo que Garza $\mathrm{R}^{5}$ afirma que un estilo de aprendizaje está relacionado con las conductas que sirven como indicadores de la manera en que aprendemos y nos adaptamos al ambiente.

Los estilos suelen ser predecibles, definen la forma de adquirir conocimientos, la estabilidad y la madurez de una persona. Cada persona tiene diversos estilos de aprender, enseñar y dirigir, aunque hay uno que en ocasiones predomina. ${ }^{5}$

Los estilos de personalidad afectan el aprendizaje y se les denomina estilos de aprendizaje y existen dos factores cuando hablamos de la manera de aprender: la percepción y el procesamiento de la información. ${ }^{5}$

De igual manera el profesor, según su personalidad, tiene un estilo de docencia, y es deseable lo que formula González $\mathrm{M},{ }^{6}$ quien expresa que el perfil del educador debe contar con 3 herramientas fundamentales: sensibilidad, flexibilidad y conocimiento, ya que actúa como un punto de referencia y de guía en el proceso de enseñanza-aprendizaje.

El reto de cada maestro es adaptar su enseñanza para satisfacer las necesidades de diferentes tipos de aprendices, sin dejar de orientar hacia el uso adecuado del nivel de sus estructuras mentales factibles de acuerdo con su edad. ${ }^{5}$

$\mathrm{Al}$ inicio de la formación, el alumno de Enfermería requiere adquirir elementos teórico-instrumentales, lo que le genera dificultades por carecer de los contenidos básicos previos, además porque debe aprender una multitud de técnicas, entre ellas la de valoración de signos vitales, la cual es el objeto de nuestro estudio, y los resultados obtenidos obligatoriamente deben integrarse al proceso enfermero, estableciendo relaciones causa-efecto sobre los resultados obtenidos con la finalidad de proporcionar un cuidado holístico.

El conocimiento sobre los signos vitales es importante cuando la enfermera utiliza el pensamiento crítico durante la fase de valoración del plan de cuidados del paciente. Para Potter, los signos vitales son indicadores importantes del estado de salud; estas medidas indican la efectividad de las funciones circulatoria, respiratoria, nerviosa y endocrina. ${ }^{7}$ Por lo tanto, la presente investigación pretende determinar las causas que influyen en el aprendizaje y los signos vitales en los alumnos del tercer semestre de la carrera de Licenciatura en Enfermería, para plantear estrategias que favorezcan el aprendizaje.

\section{Diseño metodológico}

Para realizar la presente investigación se realizó un estudio descriptivo, retrospectivo y transversal.

Las variables de estudio fueron: el nivel de destreza respecto a los signos vitales, la relación de estos parámetros en la aplicación del proceso enfermero, factores o causas que limitan o favorecen la aplicación de la técnica de signos vitales. Este estudio se define como descriptivo en la primera etapa porque busca las causas de las diversas variables que afectan el aprendizaje de los signos vitales.

Se utilizó un cuestionario para medir la percepción sobre su nivel de destreza respecto a los signos vitales; otro aspecto a estudiar es la integración de estos parámetros en la aplicación del proceso enfermero a través de casos clínicos; así, un apartado para identificar los factores o causas que limitan o favorecen la aplicación de la técnica de signos vitales.

El instrumento está conformado por preguntas abiertas, cerradas y de opción múltiple, y cuenta con cuatro partes: la primera solicita datos sociodemográficos, que incluyen edad, sexo, estado civil, número de ocasiones que cursó la materia y condición laboral; la segunda parte identifica el nivel de destreza sobre la técnica de signos vitales a través de 18 preguntas tipo likert con valores de 1 a 3 ; la tercera parte incluye casos clínicos que identifican la integración de los signos vitales en el proceso enfermero con 14 preguntas de opción múltiple. Y el último apartado identifica las causas que afectan el aprendizaje de los signos vitales a través de ocho preguntas abiertas. Para este estudio, se realizó el análisis e interpretación de los datos, utilizando programas como SPSS para el manejo de estadística descriptiva.

Se seleccionó la totalidad del universo para recabar la información del presente estudio con alumnos del Tercer Semestre de la carrera de Licenciatura en Enfermería. 
Además, mediante la Dirección de Servicios Escolares se obtuvieron las listas de los alumnos de los grupos seleccionados. También se solicitó el apoyo de los docentes del Tercer Semestre de la Carrera de Licenciatura en Enfermería de la UASLP para que nos proporcionaran tiempo para aplicar el instrumento. Se piloteó el instrumento a cinco alumnos de Octavo Semestre de la Facultad de Enfermería y se elaboró la versión final del instrumento para aplicarse a la población en estudio.

Se aplicó el instrumento durante cuatro días en diferentes horarios establecidos para cada grupo. Posteriormente se realizó el análisis e interpretación utilizando estadística descriptiva; se vaciaron los resultados en los cuadros correspondientes utilizando programas como Excel y SPSS.

\section{RESULTADOS}

El grupo de investigación incluyó 137 alumnos encuestados, de los cuales el $85 \%$ son estudiantes del sexo femenino; la media de edad es de 20 años; el estado civil de soltería en el 91\%; el $76.6 \%$ no trabaja y un $73.0 \%$ cursa por primera vez esta materia. $85.4 \%$ de los alumnos afirma que un factor a favor del aprendizaje de la técnica de signos vitales es que los profesores hagan demostraciones de la misma; $58.4 \%$ de los alumnos expresa que se realizaron ejercicios prácticos de signos vitales en el Laboratorio Propedéutico y en el aula, suficientes y pertinentes para favorecer el aprendizaje, lo que facilitó realizar esta actividad técnica con otras personas. Un factor benéfico, según el $88.3 \%$ de los alumnos, es la devolución de técnica de signos vitales por parte de los alumnos, lo cual fue útil porque los profesores retroalimentaron y corrigieron fallas.

Fue un factor útil el que los profesores retroalimentaran la técnica de signos vitales, lo cual brindó seguridad al $83 \%$ de los alumnos, porque los profesores aclararon dudas durante el procedimiento, según expresa el $92.7 \%$.

Para el $91.9 \%$ de los alumnos, la enseñanza teórica otorgada en el aula favoreció el aprendizaje de los signos vitales, porque les permitió un mejor desempeño en la práctica clínica, además de que el $82 \%$ de los alumnos señalaron qué tiempo de práctica clínica fue suficiente para reforzar esta actividad. Un factor que limita el aprendizaje de la técnica de signos vitales, según el $29 \%$ de los alumnos, es la falta de destreza o de habilidad práctica en esta técnica debido a dificultades sensitivas como la audición (29\%) y el tacto, según expresa el $21 \%$ de los alumnos.

Otra limitante son los sentimientos generados al realizar la técnica de signos vitales por primera vez; entre éstos, el temor (29\%) y la inseguridad (27\%), aunque para el $17 \%$ generó la sensación de satisfacción y alegría; a un $4 \%$ les permitió sentir seguridad al realizar la actividad.

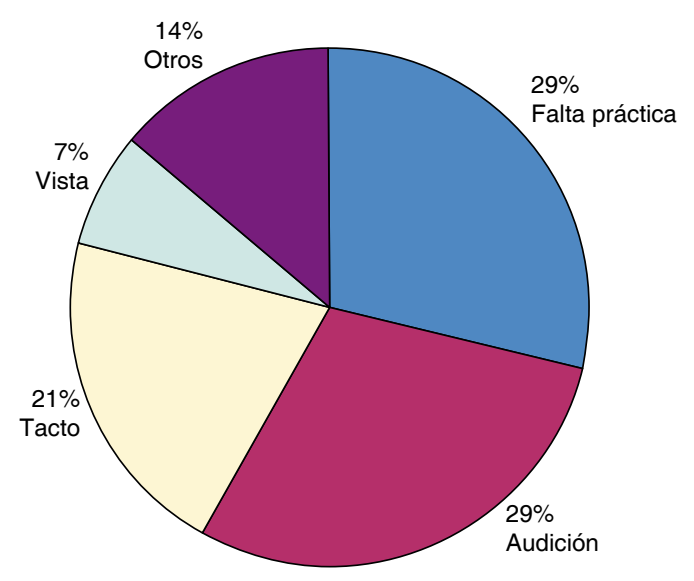

Figura 1. Causas por las que presentaron dificultades al aprender la técnica de signos vitales.

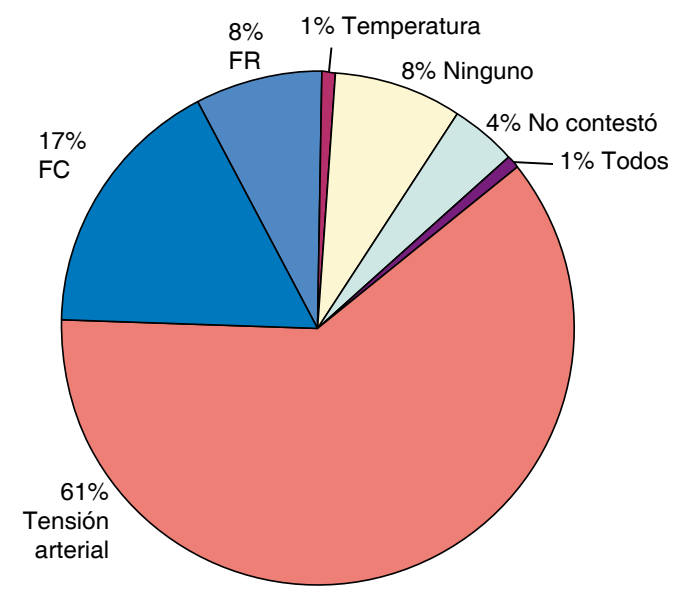

Figura 2. Signo vital más difícil de aprender.

El signo vital que les ocasionó mayor grado de dificultad fue la tensión arterial, según el $61 \%$ y las causas fueron falta de destreza o de habilidad práctica, así como dificultades con la audición, el tacto y la coordinación ojo-mano durante la medición.

El 83\% de los alumnos opinó que les resultó fácil la técnica de signos vitales, pero al interrogarlos sobre su opinión en la habilidad de integrar los parámetros obtenidos de los signos vitales para aplicar el proceso enfermero, el 59.2\% de los alumnos expresó que no hacen esta fase de juicio-clínico por falta de tiempo para realizar el proceso enfermero en los ámbitos de práctica hospitalaria o porque según el 14.7\% no lo hacen por falta de conocimientos y el $26.1 \%$ lo realiza para identificar el diagnóstico de Enfermería.

\section{DISCUSIÓN}

La investigación nos permitió identificar algunas causas que influyen en el aprendizaje de los signos vitales en alumnos 
del Tercer Semestre de la Carrera de Licenciatura en Enfermería; entre ellas se identifica como un factor favorecedor para el aprendizaje el que un $76.6 \%$ de los alumnos no trabajen, lo que es deseable, pues al ser hijos de familia en su mayoría esto les permite un mayor rendimiento escolar, presentan niveles menores de estrés a causa del dinero y tienen más tiempo para estudiar debido a que las obligaciones laborales con sus familiares pueden ser opcionales, lo que coincide con la investigación de Lara $\mathrm{A}^{8}$ sobre factores que intervienen en la trayectoria escolar de egresados de Licenciatura en Enfermería, donde un 63\% de los alumnos recibió apoyo económico familiar.

Según los alumnos, un factor benéfico para el aprendizaje es que los profesores demuestren la técnica de signos vitales (85.4\%) y que realicen ejercicios prácticos en el Laboratorio Propedéutico o en el aula (58.4\%), los cuales fueron suficientes y pertinentes para la aplicación de la técnica de signos vitales en los ámbitos de práctica, lo que confirma Davis, ${ }^{3}$ quien recomienda que para favorecer el aprendizaje de habilidades complejas o habilidades perceptivo-motoras se recomienda una demostración de la tarea o el uso de métodos audiovisuales. Según este autor, cuando se ejecuta una tarea perceptivo-motora se descubren diferentes tipos de aprendizaje dentro de la misma.

Otro aspecto útil del estudio es que los profesores retroalimentan la técnica de signos vitales, lo cual les brinda seguridad al $83 \%$ de los alumnos para ejecutarla, porque al realizar esta actividad los profesores les aclaran dudas durante el procedimiento, según expresa el 92.7\%. Este aspecto lo confirma también Davis, ${ }^{3}$ quien afirma que la retroalimentación y el conocimiento de los resultados de la tarea son extremadamente importantes porque tienen componentes tanto informativos como motivacionales.

El $91.9 \%$ de los alumnos menciona como un aspecto benéfico para el aprendizaje teórico de los signos vitales otorgado en el aula, lo que se apoya en lo encontrado por Lara $\mathrm{A},{ }^{8}$ quien muestra en su investigación sobre factores que intervienen en la trayectoria escolar de egresados de Licenciatura en Enfermería que en un 76\% los profesores siempre o casi siempre dominan los temas a enseñar. Lo que da seguridad al alumno para realizar tareas nuevas.

Diversas causas limitan el aprendizaje de la técnica de signos vitales; entre ellas están la falta de práctica o destreza en la técnica o debido a dificultades perceptivo-sensitivas-motoras. Davis $^{3}$ señala que las dificultades surgen con más frecuencia a nivel perceptivo-motor y pueden identificarse de la siguiente manera: por lateralización defectuosa, por dominante funcional izquierda o derecha mal establecida en manos, ojos y pies. Otras deficiencias de orden psicomotor pueden ser actitud defectuosa que impide la necesaria distancia para la ejecución de gestos precisos o inestabilidad psicomotriz.
También se puede caracterizar por dificultades originadas por una agitación tanto motora como del carácter, así como la hiperactividad que impide la atención y concentración. ${ }^{3}$

Otra causa es la denominada inhibición psicomotriz que origina la falta de seguridad que le impide tener la experiencia motora necesaria para el aprendizaje general. Porque los movimientos son rígidos, encogidos y representan bloqueo en todos los niveles. ${ }^{3}$ Por lo anterior, se deduce que es necesario conocer al alumno para adecuar las estrategias educativas que favorezcan el aprendizaje individual.

Respecto al temor y la inseguridad mencionados atrás, Davis $^{3}$ expresa que la ansiedad excesiva sólo agrega intranquilidad en el alumno y origina deterioro del desempeño. Y menciona que otros problemas de origen emocional pueden originar torpeza en los movimientos de las manos y los dedos que provocan lentitud.

Los signos vitales implican un conocimiento de tipo procesal donde los alumnos lleven a cabo una serie de pasos para lograr la tarea. Por lo tanto, la tensión arterial es una habilidad compleja o requiere de destrezas perceptivomotoras debido a que este procedimiento necesita estrategias instruccionales organizadas y secuenciadas, con el fin de obtener mejores resultados de aprendizaje tanto cualitativa como cuantitativamente. De ahí que los alumnos comenten que la tensión arterial sea el signo vital que mayores dificultades les originó.

La dificultad y complejidad de la técnica de signos vitales requiere que el alumno establezca relaciones causa-efecto sobre los resultados obtenidos para aplicarlos en el proceso enfermero. Esta habilidad cognitiva favorece el desarrollo del juicio clínico en el alumno que le permite identificar problemas de salud y planear las intervenciones específicas a la situación de salud del paciente a través del proceso enfermero. Lo anterior se sustenta con Garza $R,{ }^{5}$ quien comenta que el reto de cada maestro es adaptar la enseñanza para satisfacer las necesidades de diversos tipos de aprendices, sin dejar de orientar hacia el uso adecuado del nivel de sus estructuras mentales factibles de acuerdo con su edad. Con esto se quiere decir que el maestro orienta su práctica hacia los niveles de desarrollo intelectual sin olvidar que hay preferencias en la forma de aprender.

En relación a la habilidad de relacionar los parámetros de los signos vitales para aplicar el proceso enfermero, el 59.2\% de los alumnos expresa que no hacen esta integración por falta de tiempo para realizar el proceso enfermero en los ámbitos de práctica hospitalaria, el $14.7 \%$ refiere que no lo hace por falta de conocimientos y el $26.1 \%$ lo realiza para identificar el diagnóstico de Enfermería, lo que coincide con lo encontrado por Moran $\mathrm{P},{ }^{9}$ quien expresa que las dos terceras partes de los alumnos de fundamentos de Enferme- 
ría mostraron una disposición regular al proceso enfermero, apoyando esta hipótesis Ledesma $\mathrm{M},{ }^{10}$ quien comenta que los factores que influyen entre el conocimiento y aplicación del proceso enfermero son el poco tiempo y permanencia en los Servicios, la no aplicación del proceso enfermero en el campo asistencial y la no disposición del estudiante para aplicarlo. Esto contrasta con lo que menciona Gonzales $\mathrm{O},{ }^{11}$ quien señala que Wiggins y Mac Tighe (1997) afirman la existencia de un número de indicadores que señalan la comprensión del contenido teórico, entre ellos que los alumnos pueden explicar el conocimiento, predecirlo, aplicarlo o adaptarlo a nuevas situaciones; también puede hacer juicios precisos y calificados, así como conexiones con otras ideas y otros hechos. Se deduce que son múltiples las causas que limitan el aprendizaje del alumno, pero el profesor juega un papel determinante en el proceso.

Conforme a los resultados de la presente investigación, el $83 \%$ de los alumnos realiza con facilidad la técnica de signos vitales, pero sólo el $26.1 \%$ logra integrar los resultados obtenidos de estos indicadores vitales para identificar el diagnóstico de Enfermería.

\section{CONCLUSIONES}

Las causas que influyen en el aprendizaje de los signos vitales son multifactoriales; algunas están relacionadas con el estilo de aprendizaje del alumno.

De acuerdo con Garza R, ${ }^{5}$ el compromiso de los docentes es planear modelos y estrategias con los cuales el estudiante pueda desarrollar habilidades de aprendizaje que le permitan acceder por diversas vías al conocimiento. Significa ayudarle a desarrollar nuevos estilos de aprendizaje que complementen los que ya posee para capitalizar sus capacidades, a la vez que podrá compensar sus debilidades.

\section{BIBLIOGRAFÍA}

1. Tovar B. La simulación clínica: estrategia en la educación de Enfermería. Desarrollo Científico en Enfermería 2005; 13: 197-201.

2. Alfaro-Lefavre R. Pensamiento crítico y juicio clínico en enfermería. $4^{\text {ta }}$ Ed. España. Editorial Elsevier, 2009: 6: 14-15.

3. Davis R. Diseño de Sistemas de Aprendizaje. México. Editorial Trillas, 1990: 157-184, 329-347.

4. Castellanos L, Espinoza P. Aptitudes Clínicas en Egresados de Escuelas de Enfermería. Desarrollo Científico en Enfermería. 2010; 18(2): 58-63.

5. Garza R. Aprender cómo aprender. $3^{\text {ra }}$ Ed. México, Editorial Trillas, 2000; 36: 58-71.

6. González M y col. Perfil docente en el siglo XXI en la Educación Superior. Desarrollo Científico en Enfermería. 2010; 18 (4): 174-178.

7. Potter P. Fundamentos de Enfermería. $5^{\text {ta }}$ Ed. Madrid. Editorial Harcourt, 2002; 667: 683-731.

8. Lara A. Factores que intervienen en la trayectoria escolar de egresados de Licenciatura en Enfermería. Desarrollo Científico en Enfermería. 2010; 18 (2): 81-63-85.

9. Moran P. La disposición al pensamiento crítico y la organización conceptual de estudiantes de Enfermería. Desarrollo Científico en Enfermería 2005; 13 (5): 136-140.

10. Ledesma M y col. Conocimiento y aplicación del proceso de Enfermería por Estudiantes de Licenciatura. Desarrollo Científico en Enfermería 2005; 13 (3): 82-86.

11. González O. El trabajo docente, enfoques innovadores para el diseño de un curso. Primera edición. México, Editorial Trillas, 1999: 100-101. 